\title{
Efficacy of Additional Canagliflozin Administration to Type 2 Diabetes Patients Receiving Insulin Therapy: Examination of Diurnal Glycemic Patterns Using Continuous Glucose Monitoring (CGM)
}

\author{
Mihoko Matsumura - Yuki Nakatani · Seiichi Tanka • \\ Chie Aoki - Masaaki Sagara - Kazunori Yanagi · Kunihiro Suzuki • \\ Yoshimasa Aso
}

Received: March 14, 2017 / Published online: June 23, 2017

(c) The Author(s) 2017. This article is an open access publication

\section{ABSTRACT}

Introduction: The efficacy of administering a sodium-glucose cotransporter 2 inhibitor during insulin therapy has not been established. In this study, we examined its effects based on diurnal glycemic patterns using continuous glucose monitoring (CGM).

Methods: The subjects were 15 patients who had received insulin therapy for 1 year or more. A CGM device was attached to all subjects for 1 week. The administration of canagliflozin at $100 \mathrm{mg}$ was started 4 days after attachment. The mean glucose concentrations, standard deviation (SD), mean amplitude of glycemic excursions (MAGE), mean of daily difference of blood glucose (MODD), and area under the curve (AUC) $(\geq 180,<70 \mathrm{mg} \mathrm{h} / \mathrm{dL})$ after the start of administration were compared with the pretreatment values. In addition, we compared

Enhanced content To view enhanced content for this article go to http://www.medengine.com/Redeem/ 6918F06055CD8D25.

M. Matsumura $(\bowtie) \cdot$ Y. Nakatani · S. Tanka . C. Aoki - M. Sagara - K. Yanagi - K. Suzuki · Y. Aso Department of Endocrinology and Metabolism, Dokkyo Medical University, Tochigi, Japan e-mail: m-mihoko@dokkyomed.ac.jp

Y. Nakatani

Department of Diabetes and Endocrinology, Nikko Medical Center, Dokkyo Medical University,

Tochigi, Japan changes in the number of insulin units between basal and bolus insulin. Furthermore, we investigated the influence of canagliflozin on oxidative stress markers and cytokines using 8-hydroxy-2'-deoxyguanosine (8-OHdG), tumor necrosis factor- $\alpha(\mathrm{TNF}-\alpha)$, and adiponectin as parameters.

Results: The mean glucose concentrations decreased from 161.1 to $139.1 \mathrm{mg} / \mathrm{dL}(P<0.01)$. The SD decreased from 36.5 to $29.6 \mathrm{mg} / \mathrm{dL}$ $(P=0.05)$. The MAGE decreased from 89.2 to $77.4 \mathrm{mg} / \mathrm{dL} \quad(P<0.01)$, and the MODD decreased from 34.3 to $25.5 \mathrm{mg} / \mathrm{dL}(P<0.05)$. All parameters showed significant improvements in diurnal changes. AUC of $\geq 180$, i.e., the total area of blood glucose levels at or above 180 on the blood glucose curve of CGM, decreased from 339.1 to $113.6 \mathrm{mg} / \mathrm{dL}(P<0.05)$. AUC of $<70$, i.e., the total area of blood glucose levels below 70 on the blood glucose curve of CGM, slightly decreased from 1.6 to $0.3 \mathrm{mg} / \mathrm{dL}$ $(P=0.08)$. The total number of basal insulin units decreased from 128 to 76 , and that of bolus insulin decreased from 266 to 154; the dose of insulin could be markedly decreased. In addition, the mean 8-OHdG level decreased from 11.4 to $10.8 \mathrm{ng} / \mathrm{mg}$ Cre $(P<0.05)$, and the mean TNF- $\alpha$ level decreased from 2.31 to $1.79 \mathrm{pg} / \mathrm{mL}(P=0.10)$. The mean adiponectin level increased from 5.01 to $5.53 \mu \mathrm{g} / \mathrm{mL}$ $(P<0.05)$.

Conclusion: Canagliflozin improved blood glucose changes in type 2 diabetes using 
insulin. In addition, the results suggest its antioxidant actions.

Trial Registration: University Hospital Medical Information Network (UMIN no. 000019429).

Keywords: Canagliflozin; CGM (continuous glucose monitoring); Diurnal glycemic pattern; Type 2 diabetes; SGLT2 inhibitor

\section{INTRODUCTION}

The action mechanism of sodium-glucose cotransporter 2 (SGLT2) inhibitors differs from insulin secretion, and they may exhibit blood glucose-lowering effects even in patients with unfavorable blood glucose control during insulin therapy. However, few studies have examined the additional effects of SGLT2 inhibitors during insulin therapy [1-3]. Furthermore, only two studies investigated diurnal changes using continuous glucose monitoring (CGM) [4, 5]. SGLT2 inhibitors are effective in non-responders to conventional treatment or patients wishing to avoid weight gain because monotherapy with an SGLT2 inhibitor does not induce hypoglycemia [6] and these drugs decrease the body weight [7]. Therefore, the additional administration of SGLT2 inhibitors may potentiate treatment with hypoglycemia avoidance, making it possible to decrease the dose of insulin. In this study, we examined whether the additional administration of SGLT2 inhibitors during insulin therapy improves blood glucose dynamics without increasing the body weight or incidence of hypoglycemia using CGM. In addition, we investigated the influence of SGLT2 inhibitors on oxidative stress markers and cytokines.

\section{METHODS}

\section{Subjects}

The subjects were 15 patients with type 2 diabetes who had received insulin therapy for 1 year or longer, with a hemoglobin A1c (HbA1c) value of greater than $7.5 \%$. The mean age was $52.9 \pm 14.4$ years. The mean duration of diabetes was $6.6 \pm 6.7$ years. The mean duration of insulin therapy was $1.3 \pm 2.6$ years. The mean $\mathrm{HbA} 1 \mathrm{c}$ value was $10.5 \%(91 \mathrm{mmol} / \mathrm{mol})$. The mean body mass index (BMI) was $28.8 \pm 4.5 \mathrm{~kg} / \mathrm{m}^{2}$. Further information about the study population is provided in Table 1 . The study protocol was approved by the Ethical Committee of Dokkyo Medical University (no. 26100). The trial was registered with the University Hospital Medical Information Network (UMIN no. 000019429). All procedures followed were in accordance with the ethical standards of the responsible committee on human experimentation (institutional and national) and with the Helsinki Declaration of 1964 , as revised in 2013. Informed consent was obtained from all patients for being included in the study.

Table 1 Clinical characteristic of the subjects

\begin{tabular}{ll}
\hline Number (male/female) & $15(10 / 5)$ \\
Age (years) & $52.9 \pm 14.4$ \\
Body mass index $\left(\mathrm{kg} / \mathrm{m}^{2}\right)$ & $28.8 \pm 4.5$ \\
Body weight $(\mathrm{kg})$ & $75.4 \pm 15.4$ \\
Diabetic duration (years) & $6.6 \pm 6.7$ \\
Insulin therapy duration (years) & $1.3 \pm 2.6$ \\
HbAlc (\%) & $10.5 \pm 2.5$ \\
eGFR (mL/min/1.73 m $\left.{ }^{2}\right)$ & $91.6 \pm 2.5$ \\
Insulin dose (IU/day) & $26.3 \pm 16.0$ \\
Insulin therapy, $n$ (\%) & $10(66.7)$ \\
Basal plus bolus & $1(6.6)$ \\
Basal alone & $4(26.7)$ \\
Bolus alone & $1(6.6)$ \\
Glucose-lowering therapies, $n$ (\%) & $2(13.3)$ \\
Metformin & $1(6.6)$ \\
Sulfonylureas & \\
Dipeptidyl peptidase- 4 inhibitors & \\
Glucagon-like peptide-1 agonists & \\
\hline &
\end{tabular}

Date are mean \pm SD 


\section{Study Design}

This study was conducted in all cases after hospitalization. During hospitalization, the patients were on a calorie-restricted diet. The calorie-restricted diet was based on ideal body weight: [height $(\mathrm{m})]^{2} \times 22\left(\mathrm{~kg} / \mathrm{m}^{2}\right) \times 25(\mathrm{kcal} /$ $\mathrm{kg}$ /day). This is a standard diet for Japanese diabetic patients. Nutritional components are 50-60\% carbohydrate, 20-30\% lipid, and 20\% protein, every day [8]. This study was conducted after taking this calorie-restricted diet for 1 week or longer. The calorie intake remained the same from hospitalization until the end of this study. First, a CGM device (iPro2; Medtronic MiniMed, Inc.) was attached for a week. The day of CGM device attachment was regarded as day 1 . On day 7 , the device was removed. On day 4 , the administration of canagliflozin at $100 \mathrm{mg}$ was started. Besides canagliflozin, no drug was added or discontinued within this week.

The mean daily glucose concentration and standard deviation (SD) were calculated between days 1 and 7 . In addition, we calculated the mean amplitude of glycemic excursions (MAGE), mean of daily difference of blood glucose (MODD), and area under the curve (AUC) (glucose concentrations $\geq 180,<70$ ). The MAGE (mg/dL) was calculated by averaging the difference between the peak and low blood glucose levels only when the blood glucose level changed by 1 SD or more [9]. The MODD (mg/ $\mathrm{dL}$ ) was determined by averaging the absolute value of the difference in blood glucose levels at the same time for 2 days [10]. An AUC $\geq 180$ $(\mathrm{mg} \mathrm{h} / \mathrm{dL})$ is the total area of blood glucose levels at or above 180 on the blood glucose curve of CGM. An AUC $<70(\mathrm{mg} \mathrm{h} / \mathrm{dL})$ is the total area of blood glucose levels below 70 on the blood glucose curve of CGM. The MAGE and AUC were calculated every day from day 1 until day 7. Differences in the MODD between 2 days (between days 1 and 2, days 2 and 3, days 4 and 5, days 5 and 6, and days 6 and 7) were calculated.

Furthermore, the number of insulin units was compared between days 1 and 7 . In the present study, insulin was administered as a basal-bolus regimen in 10 patients, as a basal regimen in one patient, and as a bolus regimen in four patients. Therefore, we divided the total amount of insulin into basal and bolus regimens to calculate changes for these regimens.

In addition, the 8-hydroxy-2'-deoxyguanosine (8-OHdG), tumor necrosis factor- $\alpha$ (TNF- $\alpha$ ), and adiponectin levels were compared between days 1 and 7 . These cytokines were measured at $3 \mathrm{~h}$ after breakfast and at $30 \mathrm{~min}$ after bed rest.

For all cases, blood C-peptide levels (CPR: C-peptide immunoreactivity) were measured before breakfast on days 1 and 7 , and CPR indexes (CPR (ng/mL)/fasting glucose (mg/ $\mathrm{dL}) \times 100$ ) were calculated.

\section{Statistical Analysis}

The results are presented as the mean \pm standard deviation (SD). Differences between continuous variables were analyzed by paired $t$ test or Wilcoxon's matched pairs test. $P$ values less than 0.05 were considered significant. All data were analyzed with JMP7 Japanese version analytic software (SAS Japan, Tokyo, Japan).

\section{RESULTS}

As a CGM device facilitates blood glucose measurement at 5 -min intervals, 288 values per day were obtained. For all subjects, 288 sessions/day of blood glucose measurements could be performed from day 1 until day 7 .

\section{Glycemic Control}

The graph shows the mean glucose concentrations obtained on CGM. The table shows the mean daily blood glucose level, SD, MAGE, MODD, and AUC $(\geq 180,<70)$. These parameters decreased from day 4 . In addition, the data were compared between days 1 and 7 . The SD, MAGE, MODD, and AUC $(\geq 180)$ significantly decreased (Fig. 1).

\section{Dose of Insulin}

We compared the number of insulin units per day between days 1 and 7 . It decreased from 354 


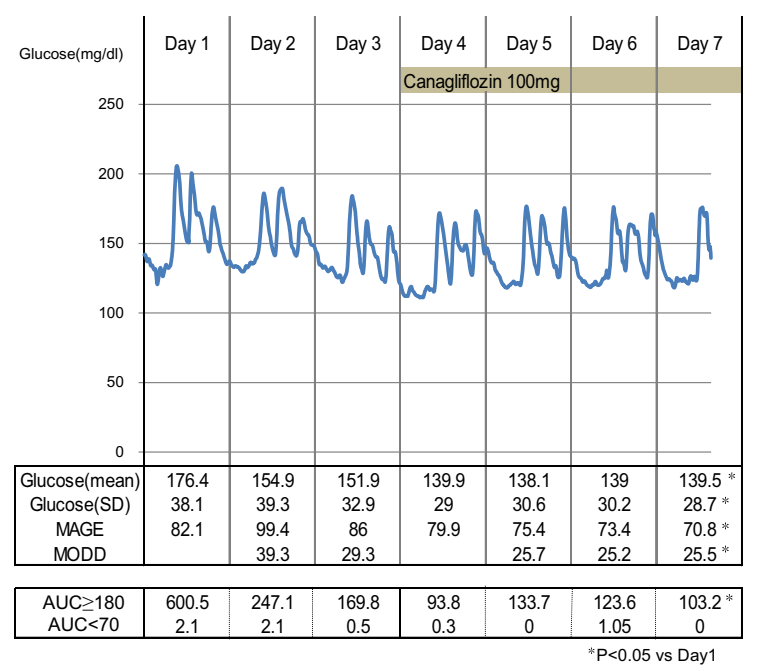

Fig. 1 Graphs show the mean glucose concentration of all subjects obtained on CGM. The table shows the mean daily glucose concentrations, SD, MAGE, MODD and area under the curve (blood glucose: $\geq 180,<70$ )

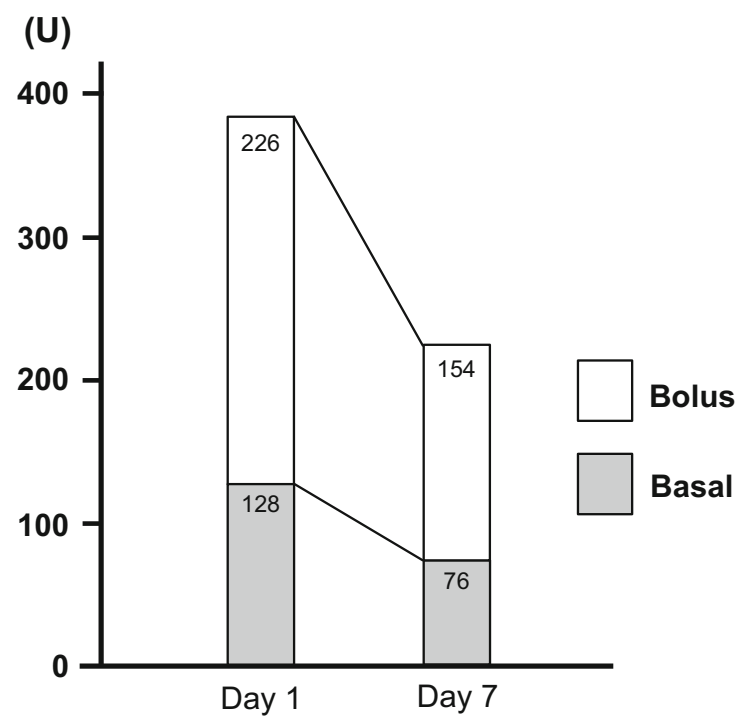

Fig. 2 The total amount of insulin in all patients was divided into basal and bolus regimens to show changes from days 1 to 7 . The number of basal insulin units decreased from 128 to 76 and that of bolus insulin from 266 to 154

to 230 units. The number of basal insulin units markedly decreased from 128 to 76 , and that of bolus insulin decreased from 266 to 154 units (Fig. 2).

\section{Oxidative Stress Markers}

Between days 1 and 7, the 8-OHdG level significantly decreased, the TNF- $\alpha$ level slightly decreased, and the adiponectin level significantly increased (Fig. 3).

\section{Function of $\beta$ Cells}

The blood C-peptide levels showed no significant change between days 1 and 7 (1.64 and $1.61 \mathrm{ng} / \mathrm{mL}$, respectively). In addition, the CPR indexes were not significantly different (1.30 and 1.46 , respectively).

\section{Safety}

The body weight significantly decreased from 76.8 to $75.4 \mathrm{~kg}$. The BMI also significantly reduced from 28.5 to $28.2 \mathrm{~kg} / \mathrm{m}^{2}$. During the study period, there were no hypoglycemic episodes. In addition, there were no adverse reactions to canagliflozin.

\section{DISCUSSION}

In this study, canagliflozin was additionally administered to patients with type 2 diabetes in whom blood glucose control by insulin therapy was unfavorable, and the blood glucose level rapidly decreased. Furthermore, the use of CGM facilitated the verification of an improvement in diurnal changes. During the observation period, there was no hypoglycemia (severe hypoglycemia), ketoacidosis, or frequent urination at night. The safety and effectiveness of the long-term (52-week) administration of canagliflozin have already been reported [11]. However, since this study involved a short (1-week) observation period, other medications and calorie intake levels were not changed in order to eliminate their effects.

In patients receiving insulin therapy, subcutaneously absorbed insulin primarily transfers into peripheral blood. As a primary issue for this therapy, glucose uptake in peripheral tissue, especially adipose tissue, exceeds that in the liver; weight gain is marked. Therefore, combination therapy with SGLT2 inhibitors is useful 

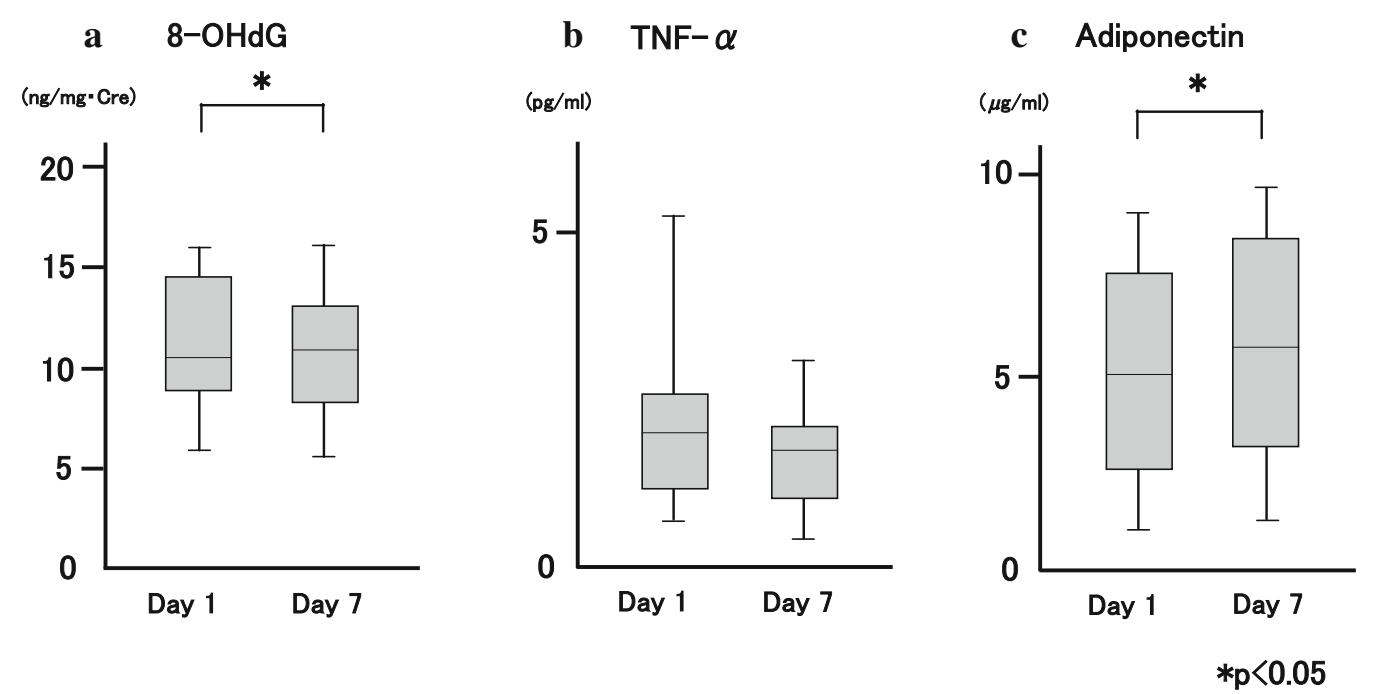

Fig. 3 Changes in the 8-OHdG, TNF- $\alpha$, and adiponectin level on days 1 to 7 of CGM are expressed as box and whisper plots. The data are expressed as the median and

in insulin-receiving patients whose body weight tends to increase.

However, SGLT2 inhibitors exhibit blood glucose-lowering effects from the first day of administration. Therefore, to prevent hypoglycemia, the dose of insulin should be rapidly decreased from the start of SGLT2 inhibitor administration. Furthermore, in this study, the number of insulin units could be markedly reduced, suggesting the blood glucose-lowering effects of canagliflozin cover a decrease in the dose of insulin. In addition, it was considered to be cost-effective [12].

The antioxidant effects of SGLT2 inhibitors may involve weight loss-related anti-inflammatory effects $[7,13,14]$, blood pressure-lowering actions $[15,16]$, an improvement in the lipid profile [15], and a decrease in the uric acid level $[16,17]$. Thus, the favorable effects of SGLT2 inhibitors other than blood glucose-reducing actions are consistent with recent diabetes treatment emphasizing not only blood glucose control but also comprehensive risk management. A meta-analysis of SGLT2 inhibitors, including canagliflozin, already demonstrated renoprotective effects [18]. Thus, SGLT2 inhibitors, including canagliflozin, may be applied as agents targeting all lifestyle-related diseases, but not solely as blood glucose-lowering agents.

The appearance of non-insulin-dependent SGLT2 inhibitors with a new action interquartile range. The 8-OHdG level significantly decreased (a), the TNF- $\alpha$ level slightly decreased $(\mathbf{b})$, and the adiponectin level significantly increased (c)

mechanism may increase the number of options for drug therapy, further advancing diabetes care. SGLT2 inhibitors are available for several procedures: monotherapy to combination therapy with other oral blood-glucose-lowering agents or insulin. Therefore, they can be selected for patients with differing background factors.

The additional administration of SGLT2 inhibitors to type 2 diabetes patients receiving insulin improved diurnal changes in the blood glucose level, decreasing the dose of insulin, suggesting that these agents are effective for insulin-related weight gain.

\section{CONCLUSION}

Canagliflozin improved blood glucose changes in type 2 diabetes patients using insulin. In addition, the results suggest its antioxidant effects. It may be useful as a second-choice drug for type 2 diabetes patients using insulin.

\section{ACKNOWLEDGEMENTS}

No funding or sponsorship was received for this study or publication of this article. The authors 
thank all the study participants and nursing stuff at our hospital. All named authors meet the International Committee of Medical Journal Editors (ICMJE) criteria for authorship for this review, take responsibility for the integrity of the work as a whole, and have given final approval for the version to be published. Mihoko Matsumura, Yuki Nakatani, and Yoshimasa Aso designed the research; Mihoko Matsumura, Yuki Nakatani, Seiichi Tanaka, Chie Aoki, Masaaki Sagara, Kazunori Yanagi, and Kunihiro Suzuki performed the research; Mihoko Matsumura, Yuki Nakatani, and Kunihiro Suzuki analyzed data; and Mihoko Matsumura, Yuki Nakatani, and Yoshimasa Aso wrote the paper. All authors read and approved the final manuscript.

Disclosures. Mihoko Matsumura, Yuki Nakatani, Seiichi Tanaka, Chie Aoki, Masaaki Sagara, Kazunori Yanagi, Kunihiro Suzuki, and Yoshimasa Aso have nothing to disclose.

Compliance with Ethics Guidelines. The study was approved by the Ethical Committee of Dokkyo Medical University (no. 26100). All procedures followed were in accordance with the ethical standards of the responsible committee on human experimentation (institutional and national) and with the Helsinki Declaration of 1964, as revised in 2013. Informed consent was obtained from all patients for being included in the study.

Data Availability. The datasets analyzed during the current study are available from the corresponding author on reasonable request.

Open Access. This article is distributed under the terms of the Creative Commons Attribution-NonCommercial 4.0 International License (http://creativecommons.org/licenses/ by-nc/4.0/), which permits any noncommercial use, distribution, and reproduction in any medium, provided you give appropriate credit to the original author(s) and the source, provide a link to the Creative Commons license, and indicate if changes were made.

\section{REFERENCES}

1. Wilding JP, Rohwedder K, Woo V, et al. Dapagliflozin in patients with type 2 diabetes receiving high doses of insulin: efficacy and safety over 2 years. Diabetes Obes Metab. 2014;16:124-36.

2. Rosenstock J, Jelasca A, Frappin G, et al. Improved glucose control with weight loss, lower insulin doses, and no increased hypoglycemia with empagliflozin added to titrated multiple daily injections of insulin in obese inadequately type 2 diabetes. Diabetes Care. 2014;37:1815-23.

3. Neal B, Perkovic V, de Zeeuw D, et al. Efficacy and safety of canagliflozin, an inhibitor of sodium-glucose cotransporter 2, when used in conjunction with insulin therapy in patients with type 2 diabetes. Diabetes Care. 2015;38:403-11.

4. Hayashi A, Takano K, Kawai S, et al. SGLT2 inhibitors provide an effective therapeutic option for diabetes complicated with insulin antibodies. Endocr J. 2016;63:187-91.

5. Perkins BA, Cherney DZ, Soleymanlou N, et al. Diurnal glycemic patterns during an 8-week open-label proof-of-concept trial of empagliflozin in type 1 diabetes. PLoS One. 2015;10:e0141085.

6. DeFronzo RA, Hompesch M, Kasichayanula S, et al. Characterization of renal glucose reabsorption in response to dapagliflozin in healthy subjects and subjects with type 2 diabetes. Diabetes Care. 2013;36:3169-76.

7. Henry RR, Murray AV, Marmolejo $\mathrm{MH}$, et al. Dapagliflozin, metformin XR, or both: initial pharmacotherapy for type 2 diabetes, a randomised controlled trial. Int J Clin Pract. 2012;66:446-56.

8. Anderson JW, Randles KM, Kendall CW, et al. Carbohydrate and fiber recommendations for individuals with diabetes: a quantitative assessment and meta-analysis of the evidence. J Am Coll Nutr. 2004;23:5-17.

9. Sevice FJ, Molnar GD, Rosevear JW, et al. Mean amplitude of glycemic excursions, a measure of diabetic instability. Diabetes. 1970;19:644-55.

10. Molnar GD, Taylor WF, Ho MM. Day-to-day variation of continuously monitored glycaemia: a further measure of diabetic instability. Diabetologia. 1972;8:342-8.

11. Qiu R, Balis D, Xie J, et al. Longer-term safety and tolerability of canagliflozin in patients with type 2 diabetes: a pooled analysis. Curr Med Res Opin. 2017;33:553-62. 
12. Karagiannis T, Bekiari E, Tsapas A. Canagliflozin in the treatment of type 2 diabetes: an evidence-based review of its place in therapy. Core Evid. 2017;12:1-10.

13. Bolinder J, Ljunggren O, Kullberg J, et al. Effects of dapagliflozin on body weight, total fat mass, and regional adipose tissue distribution in patients with type 2 diabetes mellitus with inadequate glycemic control on metformin. J Clin Endocrinol Metab. 2012;97:1020-31.

14. Wilding JP, Norwood P, T'Joen C, et al. A study of dapagliflozin in patients with type 2 diabetes receiving high doses of insulin plus insulin sensitizers: applicability of a novel insulin-independent treatment. Diabetes Care. 2009;32:1656-62.

15. Ji L, Ma J, Li H, et al. Dapagliflozin as monotherapy in drug-naive Asian patients with type 2 diabetes mellitus: a randomized, blinded, prospective phase III study. Clin Ther. 2014;36:84-100.e9.
16. Cefalu WT, Leiter LA, Yoon KH, et al. Efficacy and safety of canagliflozin versus glimepiride in patients with type 2 diabetes inadequately controlled with metformin (CANTATA-SU): 52 week results from a randomised, double-blind, phase 3 non-inferiority trial. Lancet. 2013;382:941-50.

17. Bailey CJ, Gross JL, Pieters A, et al. Effect of dapagliflozin in patients with type 2 diabetes who have inadequate glycaemic control with metformin: a randomised, double-blind, placebo-controlled trial. Lancet. 2010;375:2223-33.

18. Tang H, Li D, Zhang J, et al. Sodium-glucose co-transporter- 2 inhibitors and risk of adverse renal outcomes among patients with type 2 diabetes: a network and cumulative meta-analysis of randomized controlled trials. Diabetes Obes Metab. 2017. doi:10.1111/dom.12917. 